\title{
Optically tunable fiber grating transmission filters
}

\author{
Yicheng Lai, W. Zhang, L. Zhang, J. A. R. Williams, and I. Bennion \\ Photonics Research Group, School of Engineering and Applied Science, Aston University, Birmingham B4 7ET, UK
}

Received June 16, 2003

\begin{abstract}
We propose and demonstrate single- and multiple-passband fiber grating transmission filters that are remotely tunable by exploitation of the optical pump-induced thermal effects in Er/Yb-codoped fiber sections. A repeatable, wavelength-independent induced phase shift of $0.1 \pi / \mathrm{mW}$ is obtained without hysteresis and anisotropic effects. A transmission extinction ratio of $>23 \mathrm{~dB}$ with a 3-mW change in pump power is achieved. (C) 2003 Optical Society of America

OCIS codes: 190.4870, 050.2770, 050.5080, 060.2340.
\end{abstract}

Tunable phase-shifted fiber Bragg grating (FBG) structures are potential candidates for miniature, adjustable high-finesse transmission filters in adaptive fiber sensor applications and reconfigurable optical communication networks. ${ }^{1}$ To date, techniques for controlling the spectral characteristics of the fiber grating structure include using a thermal head, ${ }^{2}$ a piezoelectric stack, ${ }^{3}$ and local compression. ${ }^{4}$ These external tuning mechanisms generally tend to be anisotropic, which leads to undesirable birefringence effects. ${ }^{4}$ More important, the implementations of such schemes for remote operations can be complex and costly. On the other hand, resonant nonlinearity in highly doped fibers (length of a few centimeters to $\sim 2 \mathrm{~m}$ ) have been exploited to induce different index changes. ${ }^{5}$ A tunable transmission filter based on this nonlinear effect has the advantage that the actual tuning mechanism lies within the fiber itself. Such a scheme is, however, restricted by the fact the induced index change is a function of the pump and the signal wavelengths. This leads to spectral changes such as stop-band broadening ${ }^{5}$ at different pump or signal conditions and is undesirable for wideband applications.

Under continuous pumping, thermally induced phase shifts in doped fibers can exceed nonlinear phase shifts significantly. ${ }^{6}$ Nonradiative transition processes, for example, in $\mathrm{Er} / \mathrm{Yb}$-codoped fiber under 980-nm pumping, have shown large thermally induced index variations. ${ }^{7}$ Through combining such optically induced thermo-optic properties of doped fibers with passive FBG structures, practical and cost-effective means of designing miniature, all-fiber, optically remote-controlled filtering devices can be achieved. In this Letter we propose and demonstrate, for the first time to our knowledge, an optical pump-controlled remotely tunable fiber grating transmission filter configuration by utilizing the optically induced thermo-optic effect in a short section (of the order of millimeters) of doped fiber (Er/Yb-codoped fiber in this example). A tunable single phase-shifted fiber grating structure with a narrow passband $(<3 \mathrm{pm})$ is realized with a linear pump-induced phase shift of $0.1 \pi / \mathrm{mW}$. Similarly, a tunable dual phase-shifted grating structure and a multichannel passband filter with high transmission extinction ratios are illustrated. The configurations exhibit no wavelength dependence or induced anisotropic effects during operation. Since the tuning mechanism does not introduce physical strain or wear on the fiber, the spectral characteristics are highly repeatable without hysteresis. With the control pump and signal wavelength propagating within the same fiber the proposed configuration offers a simple and effective means for remote operation.

To construct an all-fiber Fabry-Perot (FP) resonator, one can splice a short section $(15 \mathrm{~mm})$ of $\mathrm{Er} / \mathrm{Yb}$-codoped fiber between two identical uniform FBGs. Without sufficient cavity gain from the doped fiber the resonator does not promote lasing operation. Instead, a multiple-passband wavelength-selective fiber grating FP transmission filter is obtained. More interestingly, a response equivalent to that of a single phase-shifted grating structure can be obtained with a much shorter section $(5 \mathrm{~mm})$ of doped fiber. If we consider the small volume of the core of the millimeter-long $\mathrm{Er} / \mathrm{Yb}$-codoped fiber $\left(7 \times 10^{-5}\right.$ to $20 \times 10^{-5} \mathrm{~mm}^{3}$ ), pump-induced thermal effects within the fiber can create a significant rise in temperature and lead to a substantial thermally induced index change, hence phase shift. The induced phase shift within the $\mathrm{Er} / \mathrm{Yb}$-codoped fiber consequently shifts the passband wavelength in the grating response. Since the thermo-optic coefficient for silica-based fiber is $>10$ times higher than the thermal expansion coefficient, ${ }^{2}$ the index change dominates the overall thermal phase shift $\Delta \phi$ in the doped fiber of length $L$ and is defined by $\Delta \phi=2 \Delta n \pi L / \lambda$, where $\lambda$ denotes the wavelength and $\Delta n$ is the index change associated with temperature. In a similar context, splicing two very short $\mathrm{Er} / \mathrm{Yb}$-codoped fiber sections between three uniform FBGs results in a structure that is equivalent to the insertion of two phase shifts in a uniform FBG, thereby yielding a transmission filter response with a wider passband peak. Optimal length ratios between the uniform FBGs in the dual phase-shifted grating structure are 1:2:1. ${ }^{8} \quad$ All these grating structures can be modeled with the transfer-matrix method. ${ }^{9}$

A 15-mm-long $\mathrm{Er} / \mathrm{Yb}$-codoped fiber (absorption at $979 \mathrm{~nm}$ of $730 \mathrm{~dB} / \mathrm{m}$ and numerical aperture of 0.22 ) was spliced between two standard single-mode fibers (SMFs) before hydrogenation. Uniform FBGs of 10 -mm length with a strength parameter of $\kappa L \sim 1.7$ were then written into the SMF on each side of the doped fiber to realize a FP multiple-passband filter. 
Similarly, a single phase-shifted grating structure was realized when a 5-mm-long $\mathrm{Er} / \mathrm{Yb}$-codoped fiber was spliced between two uniform FBGs of 16-mm length with $\kappa L \sim 2.7$. The operation and measurement setup of these grating structures is shown in Fig. 1(a). The 980-nm FBG is used to reflect the unabsorbed 980-nm pump beam to double pass the doped fiber and thereby increase the efficiency of the system. To realize a dual phase-shifted grating structure, we spliced two 4-mm-long Er/Yb-codoped fiber sections between three sections of SMF such that gratings of lengths and strengths of $8 \mathrm{~mm}$ and $\kappa L \sim 1.46,16 \mathrm{~mm}$ and $\kappa L \sim 2.40$, and $8 \mathrm{~mm}$ and $\kappa L \sim 1.46$ were written into the SMF. To equalize pump-induced phase shifts in the doped fiber sections, the bidirectional pumping scheme shown in Fig. 1(b) was adopted, wherein an adjustable coupler was used to split the $980 \mathrm{~nm}$ beam from the pump diode as required. The transmission profiles of the grating structures under different 980-nm pump powers were constantly monitored with a scanning tunable laser (resolution $1 \mathrm{pm}$ ) with an optical spectrum analyzer. The Er/Yb-codoped fiber regions were sandwiched between polystyrene foam (heat conductivity of $0.36 \mathrm{~W} / \mathrm{m}{ }^{\circ} \mathrm{C}$ ) as a means of reducing the rate of heat loss from the doped fiber while the rest of the grating structure was thermally bonded to a peltier to help keep it a constant temperature. Otherwise, passive athermal packaging, ${ }^{10}$ for example, can be used to further simplify the operation setup. Wavelength division multiplexers, used in both cases, multiplex light at 980 and $1550 \mathrm{~nm}$ through the grating structures.

A typical transmission spectrum of the single phaseshifted grating structure is shown in trace a of Fig. 2. The transmission peak has a FWHM bandwidth of $<3 \mathrm{pm}$. Above the 980-nm pump laser threshold the transmission peak shifts toward higher wavelengths proportionately with increasing pump power at a rate of $\sim 7 \mathrm{pm} / \mathrm{mW}$ as shown in Fig. 2. When the experimental data are matched to the simulation results, the pump-induced index variation in the $5-\mathrm{mm}$ section of doped fiber was found to be $1.55 \times 10^{-5} / \mathrm{mW}$. Over a pump power range between 1.5 and $11.5 \mathrm{~mW}$ the transmission peak crossed the whole stop band of the grating, corresponding to $>\pi$ rad of phase change. The transmission extinction ratio measured with a narrowband probe signal was $>16 \mathrm{~dB}$ with a $1.5-\mathrm{mW}$ change in launched pump power. This isolation can be easily improved with an increase in the strength of the uniform FBGs. Spectral profiles captured at $1.5-\mathrm{mW}$ pump power intervals indicate no wavelength-dependent phase variation. The inset in Fig. 2 shows the linear relationship between the induced phase shifts and the launched pump powers. To verify thermal phase shift as the key effect in the operation of the transmission filter, we immersed the grating structure in a water bath at $22^{\circ} \mathrm{C}$; no significant spectral changes were detected with increasing pump power.

A typical transmission profile of the dual phaseshifted grating structure is shown in trace a of Fig. 3. The widened transmission peak has a FWHM of $\sim 8 \mathrm{pm}$ and shifts toward higher wavelengths with increasing pump power, as shown in Fig. 3. The rate of wavelength shift with launched pump power was $\sim 5 \mathrm{pm} / \mathrm{mW}$ and corresponds to an induced index variation of $0.85 \times 10^{-5} / \mathrm{mW}$ in each $4-\mathrm{mm}$ section of $\mathrm{Er} / \mathrm{Yb}$-codoped fiber. The efficiency of the system is significantly affected by the greater number of sections of doped fiber as well as by the absence of recirculation of the unabsorbed $980-\mathrm{nm}$ pump beam.

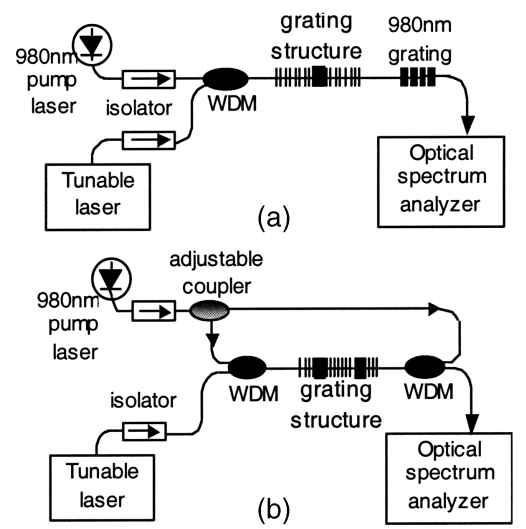

Fig. 1. Experimental setup for (a) the tunable fiber grating FP filter and the tunable single phase-shifted grating structure and (b) the tunable dual phase-shifted grating structure. WDM, wavelength division multiplexer.

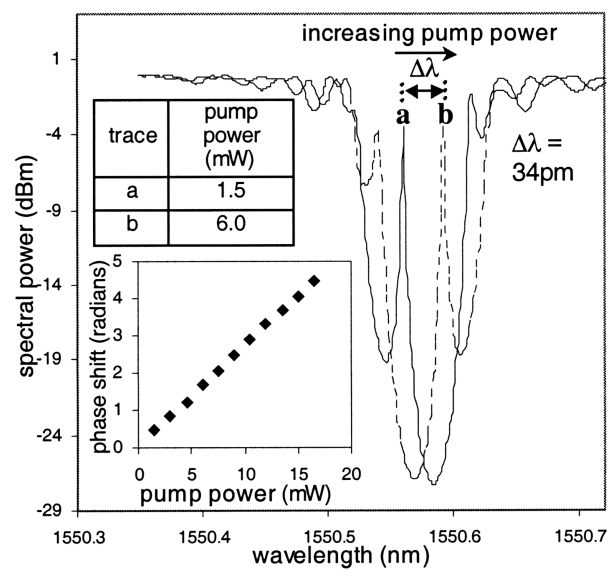

Fig. 2. Transmission profiles of the tunable single phaseshifted grating structure at 1.5 - and $6-\mathrm{mW}$ pump power. Inset, linear variation of induced phase shift with launched pump power.

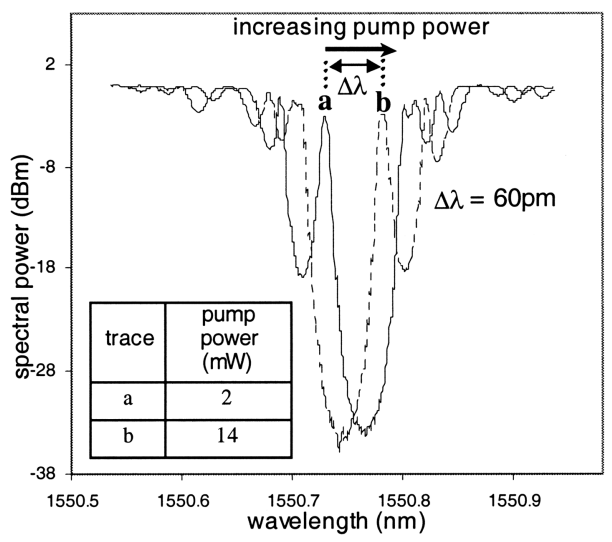

Fig. 3. Transmission profiles of the dual phase-shifted grating structure at 2- and 14-mW pump power. 


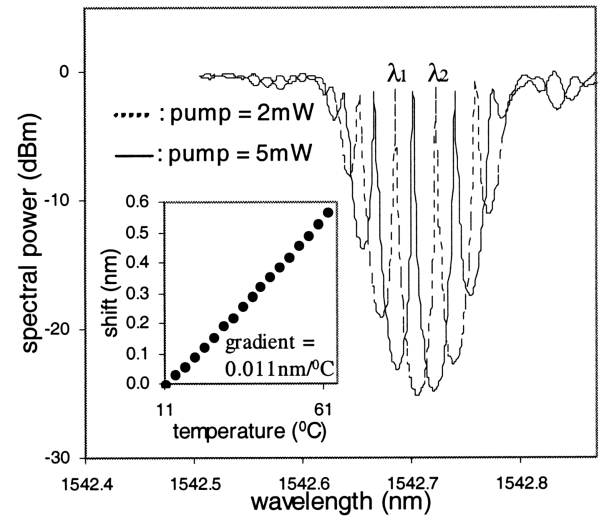

Fig. 4. Spectral profiles of the tunable FP multiplepassband filter at 2- and $5-\mathrm{mW}$ pump power. Inset, normalized Bragg wavelength shift with temperature of a uniform FBG written into the $\mathrm{Er} / \mathrm{Yb}$ fiber.

Nevertheless, over a pump increment of $23 \mathrm{~mW}$ the bandpass peak crossed the stop band of the grating, achieving a $>\pi$-rad phase shift. The transmission extinction ratio measured with a narrowband probe beam was $>21 \mathrm{~dB}$ with a $3-\mathrm{mW}$ change in pump power. Finally, the measured transmission spectra of the FP multiple-passband filter at two different pump powers are as shown in Fig. 4. Over a change in pump power of $3 \mathrm{~mW}$ the transmission passbands shift by $20 \mathrm{pm}$ (half a free spectral range), which corresponds to an induced index variation of $2.4 \times 10^{-5}$. With operation channels at $\lambda_{1}$ and $\lambda_{2}$ the transmission extinction ratio was $>23 \mathrm{~dB}$ with a $3-\mathrm{mW}$ change in pump power.

To measure the rise in temperature with pump power within the $\mathrm{Er} / \mathrm{Yb}$-codoped fiber, we inscribed a 16-mm-long uniform FBG into the $\mathrm{Er} / \mathrm{Yb}$-codoped fiber, and its Bragg wavelength shift with surrounding temperature was measured. The results shown in the inset in Fig. 4 indicate a wavelength shift of $0.011 \mathrm{~nm} /{ }^{\circ} \mathrm{C}$, which corresponds to a thermo-optic coefficient of $1.024 \times 10^{-5} /{ }^{\circ} \mathrm{C}$. Hence for the case of the single phase-shifted grating structure the pumpinduced rise in temperature within the $5-\mathrm{mm}$ section of $\mathrm{Er} / \mathrm{Yb}$-codoped fiber was $1.51^{\circ} \mathrm{C} / \mathrm{mW}$, which is of the same order of magnitude as in a previous report. ${ }^{7}$ It is important to note that the efficiency of the configuration can be improved with, for example, codoped fibers under 720-nm pumping and V-doped fibers under 906-nm pumping, ${ }^{11}$ which exhibit a light-to-heat conversion rate of $>55 \%$. Furthermore, because of the broad pump absorption bandwidth $(>20 \mathrm{~nm})$ of these doped fibers, a number of the transmission filters, in tandem with the respective FBG pump filters, can be individually addressed at different pump wavelengths. An adaptive and reconfigurable network of the transmission filters can hence be achieved.

In conclusion, optical pump-controlled, remotely tunable fiber grating transmission filters have been realized. The results highlight the proposed scheme as a potential means of implementing a simple, miniature tunable fiber grating filter.

Y. Lai's e-mail address is laiy@aston.ac.uk.

\section{References}

1. G. P. Agrawal and S. Radic, IEEE Photon. Technol. Lett. 6, 995 (1994).

2. S. Gupta, T. Mizunami, and T. Shimomura, J. Lightwave Technol. 15, 1925 (1997).

3. M. G. Xu, A. T. Alavie, R. Maaskant, and M. M. Ohn, Electron. Lett. 32, 1918 (1996).

4. C. J. S. de Matos, P. Torres, L. C. G. Valente, W. Margulis, and R. Stubbe, J. Lightwave Technol. 19, 1206 (2001).

5. M. Janos, J. Canning, and M. G. Sceats, Electron. Lett. 32, 245 (1996).

6. M. K. Davis, M. J. F. Digonnet, and R. H. Pantell, J. Lightwave Technol. 16, 1013 (1998).

7. Y. Z. Hu, H. Y. Tam, S. Y. Liu, and M. S. Demokan, IEEE Photon. Technol. Lett. 10, 1253 (1998).

8. F. Bakhti and P. Sansonetti, J. Lightwave Technol. 15, 1433 (1997)

9. M. Yamada and S. Sakuda, Appl. Opt. 26, 3474 (1987).

10. T. Iwashima, A. Inoue, M. Nishimura, and Y. Hattori, Electron. Lett. 33, 417 (1997).

11. M. K. Davis and M. J. F. Digonnet, J. Lightwave Technol. 18, 161 (2000). 